\title{
AN ASTRONOMICAL SOFTWARE DOCUMENTATION COLLEC- TION AT THE SMITHSONIAN ASTROPHYSICAL OBSERVATORY
}

\author{
Carol Rhodes, Michael Kurtz, Joyce Rey-Watson \\ Harvard-Smithsonian Center for Astrophysics \\ 60 Garden Street \\ Cambridge, Massachusetts 02138 USA
}

\begin{abstract}
We have established a library collection of documentation for software used in the reduction of astronomical data. We make some recommendations to the authors of documentation in addition to discussing the future directions of this collection. Instructions on how to contribute to and access this collection are provided.
\end{abstract}

\section{INTRODUCTION}

While working to establish this collection, we have encountered the view among authors of noncommercial astronomical software documentation that their works are too insignificant to be included in a library collection. This is understandable since library collections of documentation are a recent development. Producers of software, like other users of libraries, are essentially unaccustomed to seeing software documentation on library shelves.

This condition is unfortunate and every effort needs to be made to see that it is changed. Software is so essential to reduce and analyze the sheer volume of astronomical data collected by instruments, that without it much research would be greatly impeded. The need for software documentation is equal to that for other scientific books and, like books, documentation constitutes a major effort requiring many hours of writing and revising.

Authors of documentation should regard their efforts with the same respect they would a scientific paper or book intended for publication. Certainly, authors are encouraged to include title pages in their documentation and avoid the following omissions: the author's name, title, date of publication, the place of publication and publisher, and introductory remarks describing what the program does. Authors are also encouraged to paginate their works and to avoid title inconsistencies from cover and spine to title page. 


\section{FUTURE DIRECTIONS}

We hope that libraries at other astronomical observatories will make efforts to establish similar centralized collections of documentation. Doing so would free astronomers from the task of acquiring and keeping current on documentation, thus assisting them in their efforts to analyze data. Libraries are the best places to locate collections of documentation, since the process of acquiring documentation and subscriptions to related newsletters is like that of acquiring books and subscriptions to journals. Designating the task of acquiring and maintaining subscriptions to a library staff person would insure that the collection be kept current, thus providing an invaluable service to its users.

Through research, a collection of this type might reveal changes in the ways data are processed, manipulated and analyzed. Certainly, an archeologist of astronomy would include software as part of the astronomer's tool culture, and a collection of documentation might someday be a major resource for a thesis on the history of astronomical data reduction software. For this reason, it is imperative to collect and archive documentation for those programs no longer in use. It is also important to do the same for those older versions of software where superseding versions show significant changes.

A list of documentation currently available and information about the collection is available from

Software Documentation Collection

Smithsonian Institution Library

60 Garden St., MS 56

Cambridge, Massachusetts 02138 USA 